Journal of

Immunotoxicology http://informahealthcare.com/imt

ISSN: 1547-691X (print), 1547-6901 (electronic)

Immunotoxicol, 2015; 12(2): 188-193

(C) 2014 Informa Healthcare USA, Inc.

DOI: 10.3109/1547691X.2014.917749 informa

healthcare

RESEARCH ARTICLE

\title{
Curcumin increases gelatinase activity in human neutrophils by a p38 mitogen-activated protein kinase (MAPK)-independent mechanism
}

Francis Antoine, and Denis Girard

Laboratoire de recherche en inflammation et physiologie des granulocytes, INRS-Institut Armand-Frappier, University of Québec, Laval, Quebec, Canada

\begin{abstract}
Curcumin has been found to possess anti-inflammatory activities and neutrophils, key players in inflammation, were previously found to be important targets to curcumin in a few studies. For example, curcumin was found to induce apoptosis in neutrophils by a p38 mitogen-activated protein kinase (MAPK)-dependent mechanism. However, the role of curcumin on the biology of neutrophils is still poorly defined. To study the role of curcumin on neutrophil degranulation and to determine the role of p38 MAPK, human neutrophils were freshly isolated from healthy individuals and incubated in vitro with curcumin. Degranulation was studied at three levels: surface expression of granule markers by flow cytometry; release of matrix metallopeptidase-9 (MMP-9 or gelatinase B) enzyme into supernatants by Western blot; and gelatinase B activity by zymography. Activation of p38 MAPK was studied by monitoring its tyrosine phosphorylation levels by western blot and its role by the utilization of a pharmacological inhibitor. The results indicate that curcumin increased the cell surface expression of CD35 (secretory vesicle), CD63 (azurophilic granules), and CD66b (gelatinase granules) in neutrophils. Also, curcumin increased the release and enzymatic activity of gelatinase B in the extracellular milieu and activated p38 MAP kinase in these cells. However, in contrast to fMLP, curcumin-induced enzymatic activity and secretion of gelatinase B were not reversed by use of a p38 inhibitor. Finally, it was found that curcumin was able to enhance phagocytosis. Taken together, the results here demonstrate that curcumin induced degranulation in human neutrophils and that the increased gelatinase activity is not dependent on p38 MAPK activation. Therefore, degranulation is another human neutrophil function that could be modulated by curcumin, as well as phagocytosis.
\end{abstract}

\section{Keywords}

Degranulation, flow cytometry, inflammation, granulocytes, zymography

\section{History}

Received 3 March 2014

Revised 16 April 2014

Accepted 21 April 2014

Published online 13 June 2014 